\title{
Superiority of helical tomotherapy on liver sparing and dose escalation in hepatocellular carcinoma: a comparison study of three-dimensional conformal radiotherapy and intensity-modulated radiotherapy
}

\author{
This article was published in the following Dove Press journal: \\ OncoTargets and Therapy \\ 24 June 2016 \\ Number of times this article has been viewed
}

\author{
Qianqian Zhao',2 \\ Renben Wang ${ }^{2}$ \\ Jian Zhu \\ Linzhi Jin ${ }^{1,2}$ \\ Kunli Zhu \\ Xiaoqing $\mathrm{Xu}^{2}$ \\ Rui Feng ${ }^{2}$ \\ Shumei Jiang ${ }^{2}$ \\ Zhonghua $\mathrm{Qi}^{1,2}$ \\ Yong Yin ${ }^{2}$
}

'School of Medicine and Life Sciences, University of Jinan-Shandong Academy of Medical Sciences, ${ }^{2}$ Department of Radiation Oncology, Shandong Cancer Hospital affiliated to Shandong

University, Jinan, People's Republic of China
Correspondence: Renben Wang Department of Radiation Oncology, Shandong Cancer Hospital affiliated to Shandong University, 440 Jiyan Road, Huaiyin, Jinan, Shandong 250117 , People's Republic of China Tel/fax +8653I 6762 644I Email wangrenben@sina.cn
Background and purpose: To compare the difference of liver sparing and dose escalation between three-dimensional conformal radiotherapy (3DCRT), intensity-modulated radiotherapy (IMRT), and helical tomotherapy (HT) for hepatocellular carcinoma.

Patients and methods: Sixteen unresectable HCC patients were enrolled in this study. First, some evaluation factors of 3DCRT, IMRT, and HT plans were calculated with prescription dose at $50 \mathrm{~Gy} / 25$ fractions. Then, the doses were increased using HT or IMRT independently until either the plans reached 70 Gy or any normal tissue reached the dose limit according to quantitative analysis of normal tissue effects in the clinic criteria.

Results: The conformal index of 3DCRT was lower than that of IMRT $(P<0.001)$ or HT $(P<0.001)$, and the homogeneity index of 3DCRT was higher than that of IMRT $(P<0.001)$ or HT $(P<0.001)$. HT took the longest treatment time $(P<0.001)$. For $V_{50 \%}$ (fraction of normal liver treated to at least $50 \%$ of the isocenter dose) of the normal liver, there was a significant difference: 3 DCRT $>$ IMRT $>$ HT $(P<0.001)$. HT had a lower $D_{\text {mean }}$ (mean dose) and $V_{20}\left(V_{\mathrm{n}}\right.$, the percentage of organ volume receiving $\geq \mathrm{n}$ Gy) of liver compared with 3DCRT $(P=0.005$ and $P=0.005$, respectively) or IMRT ( $P=0.508$ and $P=0.007$, respectively). $D_{\text {mean }}$ of nontarget normal liver and $V_{30}$ of liver were higher for 3DCRT than IMRT ( $P=0.005$ and $P=0.005$, respectively) or HT ( $P=0.005$ and $P=0.005$, respectively). Seven patients in IMRT (43.75\%) and nine patients in HT (56.25\%) reached the isodose $70 \mathrm{~Gy}$, meeting the dose limit of the organs at risk.

Conclusion: HT may provide significantly better liver sparing and allow more patients to achieve higher prescription dose in HCC radiotherapy.

Keywords: hepatocellular carcinoma, radiotherapy, radiation-induced liver disease, liver sparing

\section{Introduction}

Hepatocellular carcinoma (HCC) is a major malignant disease worldwide and poses a global concern. However, previously the role of radiotherapy (RT) in HCC had been ignored because of the low tolerance dose of radiation for the whole liver, which was not satisfactory for tumor control. ${ }^{1}$ With the advanced techniques of RT, it has been possible to deliver a higher dose of radiation to part of the liver accurately without a significant dose increase in the other adjacent critical structures, especially the normal liver parenchyma. ${ }^{2}$ Now, much progress has been made in improving the therapeutic index of RT against HCC. According to the National Comprehensive Cancer Network (NCCN) practice guidelines of 2015 (revised), all tumors of HCC irrespective of the location may 
be amenable to external-beam RT (NCCN guidelines Version 1.2015). That was the first time, RT was included in NCCN as one of the comprehensive treatments for HCC.

Some studies have reported that increased radiation doses generate increased local control and survival independent of the tumor size. ${ }^{2,3}$ However, a high radiation dose to the liver is accompanied by acute and late hepatic toxicity, and radiation-induced liver disease (RILD) has been one of the most important treatment-related complications. ${ }^{1}$ A few dosimetric analyses have reported the interrelation between dose/volume parameters and the risk of RILD, and the results have been helpful in the radiation treatment plans intended to reduce RILD. ${ }^{4-7}$ Such findings encouraged additional endeavors to increase the focal liver dose for patients with unresectable intrahepatic tumors. ${ }^{3}$ The issue of how to maximize therapeutic effects by increasing the radiation dose while simultaneously sparing the adjacent normal liver is a major subject of ongoing investigations.

Modern radiation oncologists have more choice available to them than ever before for highly precise delivery of partial liver irradiation, such as three-dimensional conformal radiotherapy (3DCRT), intensity-modulated RT (IMRT), and helical tomotherapy (HT). Variations in the irradiation techniques lead to different dose distributions. HT had been shown to possess equivalent or superior capability to 3DCRT and IMRT for some malignancies, including nasopharyngeal carcinoma and endometrial cancer. ${ }^{8,9}$ But the effectiveness of HT in sparing normal liver and the potential of increasing the prescription dose for localized intrahepatic cancers specifically is unclear.

In this study, the difference between the treatment schemes (3DCRT, IMRT, and HT) in sparing normal liver was compared in the treatment of HCC. Furthermore, the study of dose increase was performed with higher tumor control and acceptable risk of complications.

\section{Patients and methods}

\section{Patient selection}

The hospital records, imaging studies, and laboratory results of patients with HCC who received RT at our hospital from January 2013 to April 2015 were retrospectively reviewed. The inclusion criteria were as follows: 1) the diagnosis of HCC made either histologically or by elevated serum $\alpha$-fetoprotein levels ( $>400 \mathrm{ng} / \mathrm{mL}$ ) with typical radiologic findings; 2) HCC with single lesion confined to the liver; 3) good general condition with Eastern Cooperative Oncology Group performance status of 2 or less and a Child-Pugh classification of A or B; and 4) whether the patients experienced transarterial chemoembolization. The study protocol was approved by the Medical Ethics Committee of the Shandong Cancer Hospital and Institute. All participants provided written informed consent.

\section{Immobilization, simulation, and target delineation}

The patients were immobilized supinely with their arms above their heads using vacuum casts. All patients used respiratory control and abdominal compression to reduce the uncertainty bias caused by respiratory movements and organ motion. Two series of axial CT (computerized tomography) images, with contrast enhancement, with $3 \mathrm{~mm}$ slick thickness including whole liver and kidneys, were acquired for each patient. The simulation CT image sets were then transferred to a Varian treatment planning system for 3DCRT and IMRT planning. After delineating all of the essential targets and organs at risk (OARs), CT datasets with structures were transferred to the tomotherapy planning system.

The gross tumor volume was defined as the primary tumor visualized in contrast-enhanced CT images. Subsequently, a $5 \mathrm{~mm}$ margin was added to create the clinical target volume. The planning target volume (PTV) was generated as the clinical target volume with a $5 \mathrm{~mm}$ radial expansion and a $10 \mathrm{~mm}$ craniocaudal expansion, taking into account patient set-up errors and respiratory liver motion. OARs including the total liver, nontarget normal liver (NTNL), stomach, kidneys, and spinal cord were also delineated for evaluation of the irradiation dose. NTNL volume was taken as the total liver volume minus PTV. All the structures were outlined by an experienced radiation oncologist.

\section{Treatment planning}

The 3DCRT and IMRT plans were all designed on a Varian Eclipse Version 8.6.23 treatment planning system. HT plans were performed with a TomoTherapy ${ }^{\circledR}$ Planning Station (TomoH ${ }^{\mathrm{TM}}$ Version 2.0.1). The calculations of 3DCRT and IMRT planning systems were performed with the Anisotropic Analytical Algorithm (Version 8.9.17) and a grid of $2.5 \mathrm{~mm}$. HT used a superposition convolution algorithm, and the calculations were done on a fine grid with a resolution of $1.875 \times 1.875 \mathrm{~mm}^{2}$. All plans were designed to achieve optimal NTNL sparing as much as possible while covering at least $95 \%$ of the PTV. Photon beams of $6 \mathrm{MV}$ or $15 \mathrm{MV}$ were used for 3DCRT and IMRT plans, and $6 \mathrm{MV}$ beams for HT plans. For 3DCRT and IMRT plans, proper number and beam orientation for the fields were chosen carefully to achieve the target goals and reduce the entering beam path 
in the normal liver according to the relationship between the PTVs and OARs. The beam setups of each individual patient in this study were designed to find the best achievable designs with NTNL sparing. The beams were directed toward the tumor along paths through the smallest liver volume to minimize the amount of normal liver exposed to even low doses of radiation. The number of fields of 3DCRT varied from 4 to 7 , and that of IMRT was 5 or 7 divided unequally. A dose rate of $300 \mathrm{MU} / \mathrm{min}$ was used for all cases. For HT plans, the iterations were based on both the target goals and dose constraints of critical structures, with the NTNL constraints as the priority. In our study, the field width and the pitch used for the HT planning optimization were $2.51 \mathrm{~cm}$ and 0.285 , respectively. The modulation factor was set to be 2.5 before optimization and adjusted during the optimization to find the optimal value. NTNLs were directionally blocked according to the relationship between PTVs and NTNLs in all HT plans. Directional blocking was used to limit the beam angles.

\section{Evaluation of treatment plans}

The starting prescribed doses were $50 \mathrm{~Gy} / 25$ fractions while meeting the OARs constraints. Then the prescription dose was increased using HT or IMRT independently at nominal $4 \%$ increments until either the plans reached 70 Gy or any normal tissue reached the dose limit according to quantitative analysis of normal tissue effects in the clinic (QUANTEC) criteria $^{10}$ and other reports..$^{7,11,12}$ The specific dose constraints were as follows: $D_{\text {mean }}$ (mean dose) of normal liver $\leq 23 \mathrm{~Gy}$; $D_{\text {mean }}$ of liver $\leq 28 \mathrm{~Gy}, V_{30}$ of liver $<50 \%$; maximum dose of stomach $\leq 54 \mathrm{~Gy}$; at least one side of kidney $\leq 23$ Gy (mean dose); and maximum dose of spinal cord $\leq 47$ Gy. The PTV doses were prescribed to cover $>95 \%$ of the PTV with no greater than $110 \%$ of the maximum point dose. To avoid any biases in the optimization and evaluation processes, normalization was set to the PTV mean dose. Having achieved these objectives, the plans were generated by the same physicist and approved by the same oncologist. For PTVs, the conformal index $(\mathrm{CI})$ is defined as $\frac{\mathrm{TV} \mathrm{PV} / V_{\mathrm{PTV}}}{V_{\mathrm{TV}} / \mathrm{TV} V_{\mathrm{PV}}}$, where $V_{\mathrm{PTV}}$ is the volume of PTV, $V_{\mathrm{TV}}$ is the volume enclosed by the prescription dose line, and $\mathrm{TV}_{\mathrm{PV}}$ is the volume of PTV within the prescribed isodose volume. ${ }^{13}$ The homogeneity index (HI) is defined as $\frac{D_{5 \%}}{D_{95 \%}}$, where $D_{5 \%}$ and $D_{95 \%}$ are the minimum doses delivered to $5 \%$ and $95 \%$, respectively, of the PTV. ${ }^{12}$ The value of $\mathrm{CI}$ ranged from 0 to 1 , and $\mathrm{HI}$ varied from infinity to 1 . The closer the value is to 1 , the better is the result. For OARs, the parameters included $D_{\text {mean }}$, the maximum dose $\left(D_{\max }\right)$, and a set of appropriate $V_{\mathrm{n}}$ (the percentage of organ volume receiving $\geq \mathrm{n}$ Gy). We evaluated each treatment plan using a dose-volume histogram and visually inspecting the isodose curves. Delivery time was recorded to assess the efficiency of treatment delivery.

\section{Statistical analysis}

The mean values (standard deviation) of the dosimetric data for the patients between the three plans were analyzed using the Friedman test. When a significant difference was found, the difference between two plans for each effect was further examined by the Wilcoxon signed-rank test. All $P$-values were two-tailed, and a value of 0.05 was considered statistically significant. All statistical analyses were performed using the SPSS Version 17.0 software (IBM Corporation, Armonk, NY).

\section{Results}

\section{Patient and tumor characteristics}

Sixteen patients with a single tumor confined to the liver were recruited for the study, and their median age was 57 years (range, 37-66 years). The ratio of males-to-females was 7:1. Ten and six patients were Child-Pugh class A and class B, respectively. All patients had received other treatments before RT. Fifteen patients were treated with transarterial chemoembolization (median number of procedures, 3; range, 1-6), and one patient was treated with radiofrequency ablation and transarterial chemoembolization. All tumors were confined to the liver without lymph node metastasis. The mean value of the volume and equivalent sphere diameter of the gross tumors were $348.57 \pm 206.11$ (range, $147.20-698.30 \mathrm{~cm}^{3}$ ) and $10.64 \pm 2.54$ (range, $6.95-15.72 \mathrm{~cm}^{3}$ ), respectively. The mean value of normal liver volume was $1,208.61 \pm 415.73 \mathrm{~cm}^{3}$ (range, 965.34-1,696.21 $\mathrm{cm}^{3}$ ). Figure 1 shows the isodose distributions in one representative patient obtained with 3DCRT, IMRT, and HT at the prescription dose of $50 \mathrm{~Gy} / 25$ fractions. Figure 2 shows the dose-volume histograms for PTV and NTNL between the three plans for the patient corresponding to Figure 1.

\section{PTV analysis and delivery time}

Table 1 shows the differences between the three techniques with regard to the dosimetric parameters of PTVs and treatment times for a prescription dose of 50 Gy. Figure 3 is a column chart of $\mathrm{CI}$ and HI for PTVs with the three modalities for all patients.

\section{OARs sparing}

Table 2 summarizes the dose statistics of the specified OARs with prescribed dose of 50 Gy. For mean value of $V_{50 \%}$ (fraction of normal liver treated to at least $50 \%$ of the 


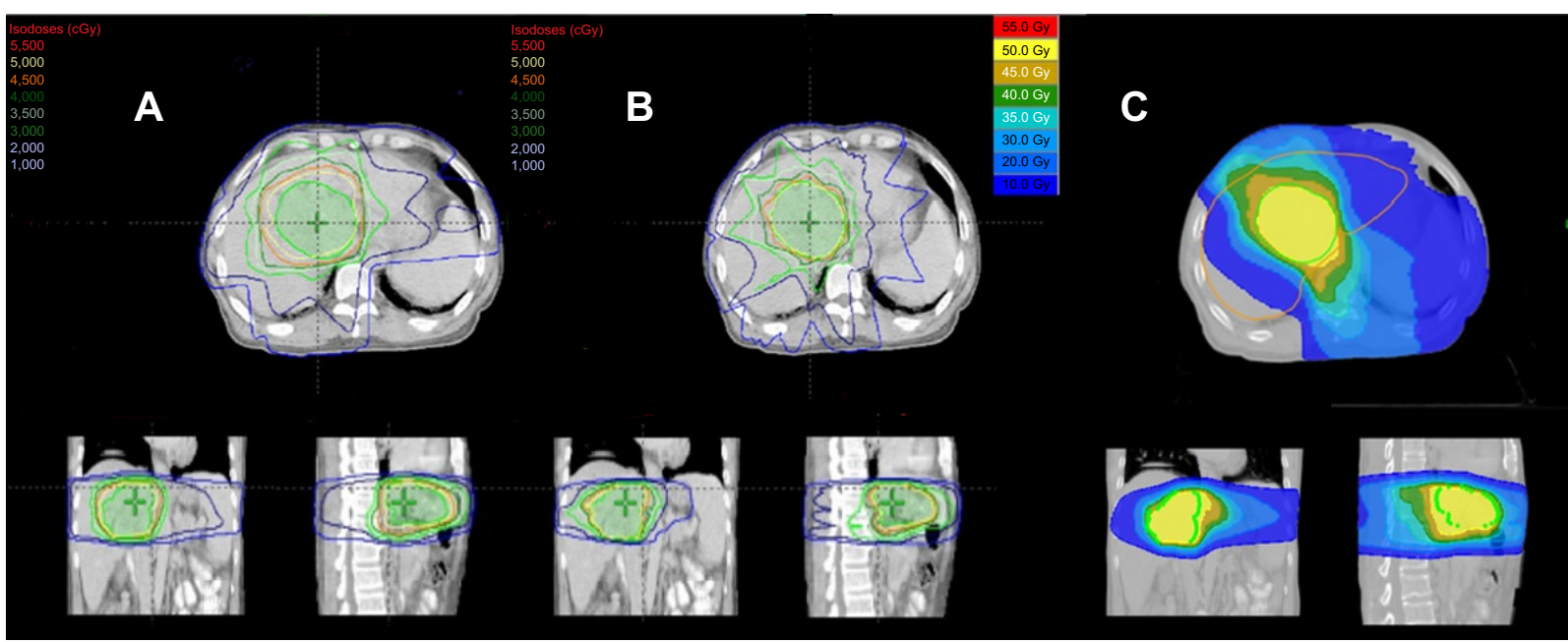

Figure I Comparison of isodose distribution of PTV and OARs in (A) 3DCRT, (B) IMRT, and (C) HT, for one representative case with a prescription dose of 50 Gy. Note: HT achieves better conformality and normal liver sparing compared with 3DCRT or IMRT.

Abbreviations: PTV, planning target volume; OARs, organs at risk; 3DCRT, three-dimensional conformal radiotherapy; IMRT, intensity-modulated radiotherapy; HT, helical tomotherapy.
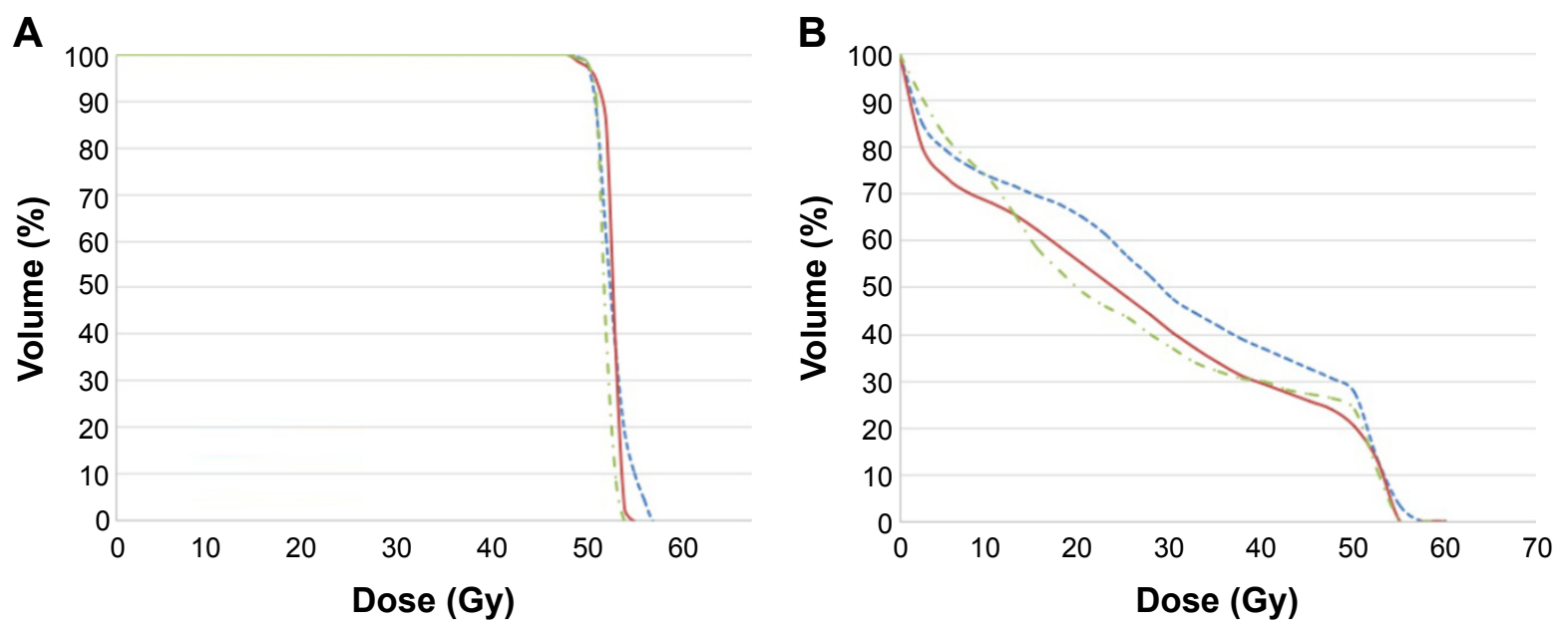

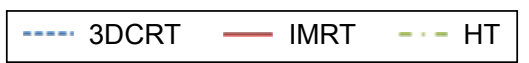

Figure 2 Comparison of the DVHs of PTVs and livers between 3DCRT, IMRT, and HT with a prescription dose of 50 Gy.

Notes: The left figure (A) shows DVHs of PTV. These three techniques produced similar homogeneity of the PTV. The right figure (B) shows DVHs of the liver. HT obtained higher low-dose distribution and the lower $V_{20}$ and $V_{30}$ compared to 3DCRT or IMRT.

Abbreviations: DVH, dose-volume histogram; PTV, planning target volume; 3DCRT, three-dimensional conformal radiotherapy; IMRT, intensity-modulated radiotherapy; $\mathrm{HT}$, helical tomotherapy.

Table I Summary of the dosimetric results for PTVs and delivery time with a prescribed dose of 50 Gy/25 fractions

\begin{tabular}{|c|c|c|c|c|c|c|c|}
\hline \multirow[t]{2}{*}{ PTV } & \multirow{2}{*}{$\begin{array}{l}\text { 3DCRT } \\
(\text { mean } \pm \text { SD) }\end{array}$} & \multirow{2}{*}{$\begin{array}{l}\text { IMRT } \\
(\text { mean } \pm \text { SD) }\end{array}$} & \multirow{2}{*}{$\begin{array}{l}\mathbf{H T} \\
(\text { mean } \pm \mathrm{SD})\end{array}$} & \multicolumn{4}{|l|}{$P$-value } \\
\hline & & & & All & 3DCRT versus IMRT & 3DCRT versus HT & IMRT versus $H T$ \\
\hline$V_{95 \%}(\%)$ & $99.90 \pm 0.23$ & $99.96 \pm 0.53$ & $99.33 \pm 0.54$ & 0.177 & - & - & - \\
\hline$V_{100 \%}(\%)$ & $80.73 \pm 1.03$ & $79.20 \pm 3.42$ & $78.34 \pm 1.21$ & 0.223 & - & - & - \\
\hline $\mathrm{Cl}$ & $0.74 \pm 0.04$ & $0.8 \mathrm{I} \pm 0.05$ & $0.82 \pm 0.04$ & $<0.001$ & $<0.001$ & $<0.001$ & 0.959 \\
\hline $\mathrm{HI}$ & $I . I I \pm 0.02$ & $1.08 \pm 0.03$ & $1.06 \pm 0.01$ & $<0.001$ & $<0.001$ & $<0.001$ & 0.286 \\
\hline Time (minutes) & $1.27 \pm 0.09$ & $3.77 \pm 1.12$ & $5.56 \pm 1.37$ & $<0.001$ & 0.005 & 0.005 & 0.013 \\
\hline
\end{tabular}

Notes: $V_{x \%}$, the volume receiving $\geq x \%$ of the prescribed dose. "-" indicates overall $P$-value among three techniques $>0.05$ and that there is no further comparison.

Abbreviations: PTV, planning target volume; 3DCRT, three-dimensional conformal radiotherapy; IMRT, intensity-modulated radiotherapy; HT, helical tomotherapy; Cl, conformal index; HI, homogeneity index; SD, standard deviation. 

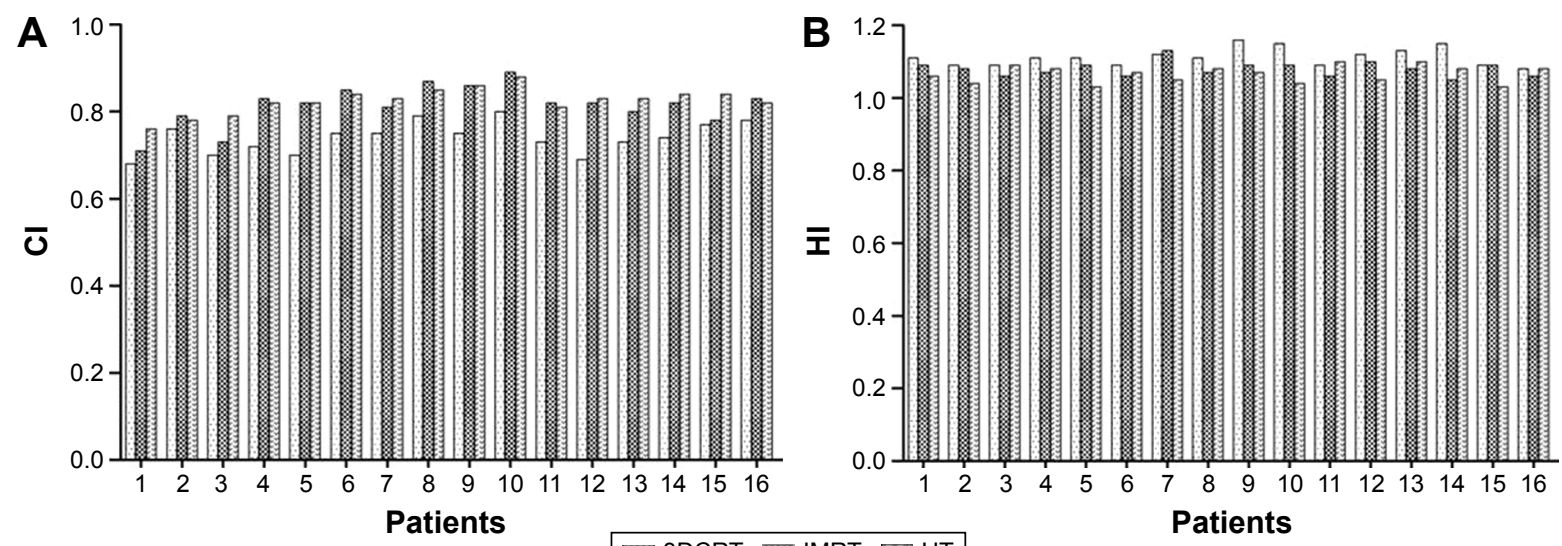

Figure 3 Column chart of conformal index $(\mathrm{Cl})$ and homogeneity index $(\mathrm{HI})$ for PTVs with three modalities for all patients.

Notes: The left figure $(\mathbf{A})$ shows the column chart of $\mathrm{Cl}$. The $\mathrm{Cl}$ of 3DCRT is lower than that of IMRT or HT. The right figure (B) shows the column chart of HI. The HI of 3DCRT is higher than that of IMRT or HT.

Abbreviations: PTV, planning target volume; 3DCRT, three-dimensional conformal radiotherapy; IMRT, intensity-modulated radiotherapy; HT, helical tomotherapy.

prescription dose) of normal liver, there was a significant difference: 3 DCRT $>$ IMRT $>$ HT $(P<0.001)$. HT had lower $D_{\text {mean }}$ and $V_{20}$ of liver compared with 3DCRT $(P=0.005$, $P=0.005)$ or IMRT $(P=0.508, P=0.007)$. For $D_{\text {mean }}$ of NTNL and $V_{30}$ of liver, 3DCRT was higher than IMRT $(P=0.005$, $P=0.005)$ or HT $(P=0.005, P=0.005)$.

\section{Dose-escalation potential of HT and IMRT}

We increased the prescribed dose for all patients using HT and IMRT independently in nominal $4 \%$ increments from the $50 \mathrm{~Gy} / 25$ fractions until either the plans reached $70 \mathrm{~Gy} / 35$ fractions or any normal tissue reached the dose limits as previously described. Seven patients in IMRT (43.75\%) and nine patients in HT (56.25\%) for whom the isodose could reach
70 Gy met the dose limit of the OARs. The increase in dose was stopped by the limit of adjacent stomach or the NTNL. For $D_{\text {mean }}$ of NTNL and $V_{30}$ of liver, HT $(15.05 \pm 0.48$ Gy and 20.32 $\pm 4.00 \mathrm{~Gy}$, respectively) was lower than IMRT (16.17 \pm 0.89 Gy and $26.32 \pm 3.38$ Gy, respectively) $(P=0.043$, $P=0.043)$. For $D_{\text {mean }}$ and $V_{20}$ of the liver, no difference was observed between the two techniques.

\section{Discussion}

Historically, RT has not played an important role in the treatment of HCC primarily due to the relatively high radiosensitivity of the liver and the risk of RILD. ${ }^{1,14}$ Several dosimetric analyses for quantifying the risk of RILD have been reported. In the study by Cheng et al, the mean dose to normal liver of patients with RILD was significantly

Table 2 Comparison of dosimetric parameters of normal organs at risk with a prescribed dose of $50 \mathrm{~Gy} / 25$ fractions

\begin{tabular}{|c|c|c|c|c|c|c|c|}
\hline \multirow[t]{2}{*}{ Structure } & \multirow{2}{*}{$\begin{array}{l}\text { 3DCRT } \\
(\text { mean } \pm \text { SD) }\end{array}$} & \multirow{2}{*}{$\begin{array}{l}\text { IMRT } \\
(\text { mean } \pm \text { SD })\end{array}$} & \multirow{2}{*}{$\begin{array}{l}\text { HT } \\
(\text { mean } \pm S D)\end{array}$} & \multicolumn{4}{|l|}{$P$-value } \\
\hline & & & & Overall & 3DCRT vs IMRT & 3DCRT vs HT & IMRT vs HT \\
\hline NTNL $V_{50 \%}(\%)$ & $26.06 \pm 11.28$ & $18.67 \pm 8.96$ & $15.05 \pm 8.80$ & $<0.001$ & 0.005 & 0.005 & 0.007 \\
\hline Liver $D_{\text {mean }}(G y)$ & $21.49 \pm 6.33$ & $18.99 \pm 5.80$ & $18.06 \pm 5.45$ & $<0.001$ & 0.005 & 0.005 & 0.508 \\
\hline NTNL $D_{\text {mean }}(G y)$ & $15.57 \pm 5.04$ & $12.48 \pm 3.97$ & $11.21 \pm 3.69$ & $<0.001$ & 0.005 & 0.005 & 0.047 \\
\hline Liver $V_{5}(\%)$ & $72.16 \pm 9.55$ & $66.22 \pm 13.01$ & $68.27 \pm 11.86$ & 0.014 & 0.005 & 0.285 & 0.445 \\
\hline Liver $V_{10}(\%)$ & $57.39 \pm 12.88$ & $52.62 \pm|2.7|$ & $50.79 \pm 13.78$ & 0.150 & - & - & - \\
\hline Liver $V_{20}(\%)$ & $40.37 \pm|7.9|$ & $37.07 \pm 13.67$ & $31.33 \pm 13.75$ & $<0.001$ & 0.139 & 0.005 & 0.007 \\
\hline Liver $V_{30}(\%)$ & $31.09 \pm 15.76$ & $25.36 \pm|4.5|$ & $23.18 \pm|2.8|$ & $<0.001$ & 0.005 & 0.005 & 0.203 \\
\hline Stomach $D_{\text {mean }}(G y)$ & $|4.24 \pm| \mid .83$ & $12.31 \pm 10.59$ & $15.14 \pm 12.12$ & 0.122 & - & - & - \\
\hline Lt kidney $D_{\text {mean }}(G y)$ & $0.64 \pm 0.58$ & $0.67 \pm 0.67$ & $1.37 \pm 1.46$ & 0.002 & 0.610 & 0.005 & 0.007 \\
\hline Rt kidney $D_{\text {mean }}(G y)$ & $1.54 \pm 2.42$ & $1.45 \pm 2.63$ & $2.33 \pm 3.05$ & $<0.001$ & 0.074 & 0.007 & 0.005 \\
\hline Spinal cord $D_{\max }(G y)$ & $20.5 I \pm I I .03$ & $|4.60 \pm| 4.2 \mid$ & $26.94 \pm 9.18$ & 0.082 & - & - & - \\
\hline
\end{tabular}

Notes: $V_{50 \%}$, fraction of normal liver treated to at least $50 \%$ of the isocenter dose; $V_{n}$, the percentage of organ volume receiving $\geq n$ Gy; $D_{\text {mean }}$, the mean dose for organ; $D_{\text {max }}$, the maximum dose for organ. "-" indicates overall $P$-value among three techniques $>0.05$ and that there is no further comparison.

Abbreviations: 3DCRT, three-dimensional conformal radiotherapy; IMRT, intensity-modulated radiotherapy; HT, helical tomotherapy; NTNL, nontarget normal liver; Lt, left; Rt, right; SD, standard deviation. 
higher than those without (25.04 Gy vs $19.65 \mathrm{~Gy}, P=0.02$ ), and it was the most important determinant for RILD. ${ }^{5}$ The University of Michigan Medical Center showed that the mean dose to normal liver associated with a $5 \%$ risk of classic RILD was 28 Gy for primary liver tumor. ${ }^{7}$ Additionally, Cheng et $\mathrm{al}^{4}$ suggested that $V_{30}<42 \%$ could avoid RILD, and Kim et $\mathrm{al}^{6}$ reported that $V_{30}$ was a significant parameter in patients treated with conventional fractionated RT. Liang et al showed that $V_{20}$ was an important parameter in patients treated with hypofractionated RT (4-6 Gy/fraction). ${ }^{15}$ In the current study, the value of the mean dose of NTNL of HT was lower than for IMRT $(P=0.047)$ or 3DCRT $(P=0.005)$. The mean dose and $V_{30}$ of liver for 3DCRT was higher than for IMRT ( $P=0.005, P=0.005)$ or HT $(P=0.005, P=0.005)$. There was significantly lower $V_{20}$ of liver for HT compared with 3DCRT $(P=0.005)$ or IMRT ( $P=0.007)$. Theoretically, based on the correlation between these dosimetric parameters and RILD, the advantage of liver sparing of HT over 3DCRT and IMRT is clear. In other words, HT could be considered as a superior choice for HCC patients in terms of normal liver sparing compared with 3DCRT or IMRT.

Evidence of a dose-response relationship has been shown. A higher prescribed dose showed a higher survival rate. For example, Dawson et al reported that patients treated with $>70$ Gy had improved in-field local control than those who received $<70$ Gy (median time to in-RT field progression, 22 months vs 9 months; $P<0.05) .{ }^{3}$ The goal of RT for $\mathrm{HCC}$ is to maximize therapeutic effects by increasing the radiation dose while sparing the adjacent normal organs. $V_{50 \%}$ of normal liver had been used as the indicator for prescribing the different dose levels. Based on the University of Michigan guideline, ${ }^{16} V_{50 \%}$ of normal liver is divided into three intervals: if the percentage of nontumor liver volume receiving $50 \%$ of the isocenter dose was $<33 \%$, the total dose was increased to $66-72.6 \mathrm{~Gy}$; if the percentage was $33 \%-66 \%$, the dose was 48-52.8 Gy; if the percentage was $>66 \%$, the total dose was $36 \mathrm{~Gy}$. In our study, the mean values of $V_{50 \%}$ of normal liver were $26.06 \% \pm 11.28 \%$ for 3 DCRT, $18.67 \% \pm 8.96 \%$ for IMRT, and $15.05 \% \pm 8.80 \%$ for HT. In accordance with the Michigan University guideline, there were nine patients in 3DCRT, eleven patients in IMRT, and 13 patients in HT who could receive the 66-72.6 Gy prescribed dose level, as shown in Table 3. This indicated that HT could allow more patients to receive a higher prescribed dose level than IMRT or 3DCRT. These data are consistent with the results of our study. Consequently, a higher prescribed dose can be given when HT is selected. In addition, from our dosimetric data, we could see that the advantage of liver sparing of HT compared with IMRT remains at the higher dose level.
Table 3 Liver tumor radiation dose prescription guideline by $V_{50 \%}$ for the patients using different radiotherapy modalities

\begin{tabular}{lllll}
\hline $\mathbf{V}_{\mathbf{5 0} \%}$ & Total dose (Gy) & $\begin{array}{l}\text { 3DCRT } \\
\text { patients } \\
(\mathbf{n})\end{array}$ & $\begin{array}{l}\text { IMRT } \\
\text { patients } \\
(\mathbf{n})\end{array}$ & $\begin{array}{l}\text { HT } \\
\text { patients } \\
(\mathbf{n})\end{array}$ \\
\hline$<33$ & Range: $66-72.6$ (focal liver) & 9 & $1 \mathrm{I}$ & 13 \\
$33-66$ & Range: $48-52.8$ (focal liver) & 7 & 5 & 3 \\
$>66$ & Mean: 36 (whole liver) & 0 & 0 & 0 \\
\hline
\end{tabular}

Note: $V_{50 \%}$, fraction of normal liver treated to at least $50 \%$ of the isocenter dose. Abbreviations: 3DCRT, three-dimensional conformal radiotherapy; IMRT, intensity-modulated radiation therapy; $\mathrm{HT}$, helical tomotherapy.

To simplify plan intercomparisons, all our HT plans were assigned with the same values of jaw width $(2.5 \mathrm{~cm})$ and pitch $(0.287)$. These parameters were chosen based on a combination of recommendations from other tomotherapy users and our own institutional experience. Moldovan et al reported that these parameter differences may not be radiobiologically meaningful based on the calculations of tumor control probabilities and normal tissue complication probabilities. ${ }^{17}$ In the present study, we protected the NTNL with a directional blocking technique that is unique for HT. Directional blocking allows a beam to pass through a given structure only after passing through the PTV such that the structure receives the exit dose but not the entry dose. ${ }^{18}$ In addition, HT shows superior dose gradient and distribution because the dose was delivered rotationally with higher intensity modulation. ${ }^{19}$ With these advantages, HT could achieve better liver sparing in patients with HCC, as shown in Figure 1. For irregular-shaped targets, the uniformity of dose distribution and sparing of normal liver are limited by 3DCRT. Our results show that the HI of 3DCRT was higher than that of IMRT $(P<0.001)$ or HT $(P<0.001)$, and the HI of HT was lower than that of IMRT though there was no statistical significance. Because of its $360^{\circ}$ beam arrangement, HT planning can provide better dose distribution than IMRT or 3DCRT. ${ }^{20}$ These indicate that HT can achieve greater dose heterogeneity to meet the more complicated tumor shapes. This advantage is more obvious in RT with complex targets; Han et al had shown improvements in homogeneity with HT in intracranial situations with irregular-shaped targets. ${ }^{19}$ Our results are, however, at variance with those of a previous study. Song et al reported that HT could reduce the probability of radiation-induced hepatic toxicity compared to fixed-beam IMRT. However, they suggested the parameter $V_{15}$ of the normal liver for predicting the probability of radiation-induced hepatic toxicity. ${ }^{21}$ Not only the parameters they suggested but also the definitions of damage were different from ours. Hsieh et $\mathrm{al}^{22}$ reported that $\mathrm{HT}$ has better potential than coplanar IMRT for HCC patients with portal vein thrombosis. They showed the superiority of HT based on the lower of $V_{30}$ in the liver ( $21 \%$ in coplanar IMRT, $17 \%$ in tomotherapy), and the data are consistent with the results of our study. 
There are some limitations with regard to our results. Any intrinsic difference in the dose calculation algorithms performed by two different treatment planning systems might produce different results. Moreover, only 16 patients were enrolled in our dosimetric analysis, which is a small number for statistical analysis. To reduce the motion of liver in RT, we used respiratory control and abdominal compression. Furthermore, we could have performed four-dimensional computed tomography using an external respiratory signal to acquire different phases of CT images for these patients to evaluate the motion of liver. ${ }^{23}$

\section{Conclusion}

Compared with 3DCRT and IMRT, HT provides significantly better liver sparing considering the lower $V_{20}, V_{30}$, or the mean dose of liver in terms of the risk of RILD for HCC. Moreover, HT is an advantageous technique for increasing the prescribed dose so that it can allow more patients to receive a higher prescribed dose with higher tumor control and acceptable risk of complications in the RT of HCC.

\section{Disclosure}

The authors report no conflicts of interest in this work.

\section{References}

1. Lawrence TS, Robertson JM, Anscher MS, Jirtle RL, Ensminger WD, Fajardo LF. Hepatic toxicity resulting from cancer treatment. Int J Radiat Oncol Biol Phys. 1995;31(5):1237-1248.

2. Park HC, Seong J, Han KH, Chon CY, Moon YM, Suh CO. Doseresponse relationship in local radiotherapy for hepatocellular carcinoma. Int J Radiat Oncol Biol Phys. 2002;54(1):150-155.

3. Dawson LA, McGinn CJ, Normolle D, et al. Escalated focal liver radiation and concurrent hepatic artery fluorodeoxyuridine for unresectable intrahepatic malignancies. J Clin Oncol. 2000;18(11):2210-2218.

4. Cheng JC, Wu JK, Huang CM, et al. Radiation-induced liver disease after radiotherapy for hepatocellular carcinoma: clinical manifestation and dosimetric description. Radiother Oncol. 2002;63(1):41-45.

5. Cheng JC, Wu JK, Huang CM, et al. Radiation-induced liver disease after three-dimensional conformal radiotherapy for patients with hepatocellular carcinoma: dosimetric analysis and implication. Int $J$ Radiat Oncol Biol Phys. 2002;54(1):156-162.

6. Kim TH, Kim DY, Park JW, et al. Dose-volumetric parameters predicting radiation-induced hepatic toxicity in unresectable hepatocellular carcinoma patients treated with three-dimensional conformal radiotherapy. Int J Radiat Oncol Biol Phys. 2007;67(1):225-231.

OncoTargets and Therapy

\section{Publish your work in this journal}

OncoTargets and Therapy is an international, peer-reviewed, open access journal focusing on the pathological basis of all cancers, potential targets for therapy and treatment protocols employed to improve the management of cancer patients. The journal also focuses on the impact of management programs and new therapeutic agents and protocols on
7. Liang SX, Zhu XD, Xu ZY, et al. Radiation-induced liver disease in three-dimensional conformal radiation therapy for primary liver carcinoma: the risk factors and hepatic radiation tolerance. Int $J$ Radiat Oncol Biol Phys. 2006;65(2):426-434.

8. Lee TF, Fang FM, Chao PJ, Su TJ, Wang LK, Leung SW. Dosimetric comparisons of helical tomotherapy and step-and-shoot intensitymodulated radiotherapy in nasopharyngeal carcinoma. Radiother Oncol. 2008;89(1):89-96.

9. Yang R, Xu S, Jiang W, Xie C, Wang J. Integral dose in three-dimensional conformal radiotherapy, intensity-modulated radiotherapy and helical tomotherapy. Clin Oncol (R Coll Radiol). 2009;21(9):706-712.

10. Marks LB, Yorke ED, Jackson A, et al. Use of normal tissue complication probability models in the clinic. Int J Radiat Oncol Biol Phys. 2010;76(3 suppl):S10-S19.

11. Dawson LA, Ten Haken RK. Partial volume tolerance of the liver to radiation. Semin Radiat Oncol. 2005;15(4):279-283.

12. Emami B, Lyman J, Brown A, et al. Tolerance of normal tissue to therapeutic irradiation. Int J Radiat Oncol Biol Phys. 1991;21(1):109-122.

13. Paddick I. A simple scoring ratio to index the conformity of radiosurgical treatment plans. Technical note. J Neurosurg. 2000;93(suppl 3): 219-222.

14. Lansing AM, Davis WM, Brizel HE. Radiation hepatitis. Arch Surg. 1968;96(6):876-882.

15. Liang SX, Huang XB, Zhu XD, et al. Dosimetric predictor identification for radiation-induced liver disease after hypofractionated conformal radiotherapy for primary liver carcinoma patients with Child-Pugh Grade A cirrhosis. Radiother Oncol. 2011;98(2):265-269.

16. Robertson JM, Lawrence TS, Dworzanin LM, et al. Treatment of primary hepatobiliary cancers with conformal radiation therapy and regional chemotherapy. J Clin Oncol. 1993;11(7):1286-1293.

17. Moldovan M, Fontenot JD, Gibbons JP, et al. Investigation of pitch and jaw width to decrease delivery time of helical tomotherapy treatments for head and neck cancer. Med Dosim. 2011;36(4):397-403.

18. Patel RR, Becker SJ, Das RK, Mackie TR. A dosimetric comparison of accelerated partial breast irradiation techniques: multicatheter interstitial brachytherapy, three-dimensional conformal radiotherapy, and supine versus prone helical tomotherapy. Int J Radiat Oncol Biol Phys. 2007;68(3):935-942.

19. Han C, Liu A, Schultheiss TE, Pezner RD, Chen YJ, Wong JY. Dosimetric comparisons of helical tomotherapy treatment plans and step-and-shoot intensity-modulated radiosurgery treatment plans in intracranial stereotactic radiosurgery. Int J Radiat Oncol Biol Phys. 2006; 65(2):608-616.

20. Mackie TR. History of tomotherapy. Phys Med Biol. 2006;51(13): R427-R453.

21. Song JH, Son SH, Kay CS, Jang HS. Reducing the probability of radiation-induced hepatic toxicity by changing the treatment modality from helical tomotherapy to fixed-beam intensity-modulated radiotherapy. Oncotarget. 2015;6(32):33952-33960.

22. Hsieh CH, Liu CY, Shueng PW, et al. Comparison of coplanar and noncoplanar intensity-modulated radiation therapy and helical tomotherapy for hepatocellular carcinoma. Radiat Oncol. 2010;5:40.

23. Underberg RW, Lagerwaard FJ, Cuijpers JP, Slotman BJ, van Sornsen de Koste JR, Senan S. Four-dimensional CT scans for treatment planning in stereotactic radiotherapy for stage I lung cancer. Int $J$ Radiat Oncol Biol Phys. 2004;60(4):1283-1290.

patient perspectives such as quality of life, adherence and satisfaction. The manuscript management system is completely online and includes a very quick and fair peer-review system, which is all easy to use. Visit http://www.dovepress.com/testimonials.php to read real quotes from published authors. 